\title{
Is there room in quantum ontology for a genuine causal role of consciousness?
}

\author{
Pylkkänen, Paavo
}

Palgrave Macmillan

2017

Pylkkänen , P 2017, Is there room in quantum ontology for a genuine causal role of consciousness? in A Khrennikov \& E Haven (eds), The Palgrave Handbook of Quantum Models in Social Science : Applications and Grand Challenges . Palgrave Macmillan, London , pp. 293-317 . https://doi.org/10.1057/978-1-137-49276-0_14

http://hdl.handle.net/10138/310681

https://doi.org/10.1057/978-1-137-49276-0_14

acceptedVersion

Downloaded from Helda, University of Helsinki institutional repository.

This is an electronic reprint of the original article.

This reprint may differ from the original in pagination and typographic detail.

Please cite the original version. 


\title{
Is there room in quantum ontology for a genuine causal role of consciousness?
}

\section{Paavo Pylkkänen 1,2}

1 Academy of Finland Center of Excellence in the Philosophy of the Social Sciences (TINT) and Department of Philosophy, History, Culture and Art Studies, P.O. Box 24 (Unioninkatu 40 A), FI00014 University of Helsinki, Finland, E-Mail: paavo.pylkkanen@helsinki.fi, Tel: +358504154822

2 Department of Cognitive Neuroscience and Philosophy, School of Biosciences, University of Skövde, P.O. Box 408, SE-541 28 Skövde, Sweden, E-Mail: paavo.pylkkanen@his.se

\begin{abstract}
Western philosophy and science have a strongly dualistic tradition regarding the mental and physical aspects of reality, which makes it difficult to understand their possible causal relations. In recent debates in cognitive neuroscience it has been common to claim on the basis of neural experiments that conscious experiences are causally inefficacious. At the same time there is much evidence that consciousness does play an important role in guiding behavior. This article explores whether a new way of understanding the causal role of mental states and consciousness could be provided by the ontological interpretation of the quantum theory (Bohm and Hiley 1987, 1993). This interpretation radically changes our notion of matter by suggesting that a new type of active information plays a causal role at the quantum level of reality. The strategy of the paper will thus be to consider to what extent the alleged causal powers of consciousness involve information, and then move on to consider whether information in (conscious) mental states can be connected to the information at the level of quantum physics. In this way we are sketching how quantum theory might help to throw light upon one of the Grand Challenges facing the social sciences and the humanities, namely the question of whether consciousness plays any genuine causal role in the physical world.
\end{abstract}

Keywords: function of consciousness; mental causation; epiphenomenalism; active information; Bohm; Hiley.

"It may be said, indeed, that without bones and muscles and the other parts of the body I cannot execute my purposes. But to say that I do as I do because of them, and that this is the way in which the mind acts, and not from the choice of the best, is a very careless and idle mode of speaking. I wonder that they cannot distinguish the cause from the condition, which the many, feeling about in the dark, are always mistaking and misnaming." Plato, The Phaedo 


\section{Introduction}

Does consciousness have causal powers? Does it make a difference to the effects of information processing, whether or not the system is conscious of a given item of information? Are our actions at least sometimes determined by our conscious free will? Since Libet's (1985) work on the neuroscience of free will, the notion that the conscious will is not the original determinant of action has won increasing support. For example, Max Velmans's (1991) work suggests that consciousness “...is neither necessary for any type of mental ability nor does it occur early enough to act as a cause of the acts or processes typically thought to be its effects" (Van Gulick 2014: 36). The radical upshot of this line of thinking is the claim that "...the sorts of mental abilities that are typically thought to require consciousness can all be realized unconsciously in the absence of the supposedly required self-awareness" (ibid.: 36). In Libet's famous studies conscious self-awareness is present, but Van Gulick notes that many claim that it occurs too late to be the cause of the relevant actions: "Self-awareness or meta-mental consciousness according to these arguments turns out to be a psychological after-effect rather than an initiating cause, more like a post facto printout..." (ibid.: 36). Van Gulick adds, however, that the arguments are controversial and that many theorists regard the empirical data as no real threat to the causal status of consciousness (for a recent discussions of the issue from various viewpoints, see e.g. Pockett et al. Eds. 2006).

But how are we to understand the causal status of consciousness? In philosophy of mind there has been a long debate about the problem of mental causation. Many philosophers assume that consciousness is in some sense a non-physical property. But this immediately gives rise to the problem of understanding how something non-physical could possibly influence something physical. A key idea to be explored in this paper is that the ontological interpretation of quantum theory might throw new light upon this perennial issue. This interpretation suggests that a new type of active information is playing a key causal role in physical processes at the quantum level. Now, when one examines the various suggestions about the putative causal powers of consciousness, many of them refer to the role of information, in one way or another. This then suggests a strategy for the present paper. We will first consider how the various suggestions about the causal status of consciousness involve information; we will then ask whether such information in mental and conscious states could be connected to information at the quantum level. This way we could begin understand mental causation, and the causal role of conscious experiences in particular, in a new way. Of course, this is a big and difficult issue and we can only sketch the solution in a short article. However, even this sketch will hopefully illustrate the great potential of quantum theory when trying to meet some of the Grand Challenges facing the social sciences. 


\section{Van Gulick and Revonsuo on the causal efficacy of consciousness}

In his useful review of the suggestions about the causal role of consciousness Van Gulick (2014: 34-42) says that consciousness is thought to provide the organism with a) more flexible control; b) better social coordination; c) more integrated representation; d) more global informational access; e) increased freedom of will; and f) intrinsic motivation. In this section I will briefly explicate these (as well as some of Revonsuo's (2006) related ideas) and then, in the next section, discuss how they connect with the notion of information. Note that the aim in this paper is not to critically evaluate these suggestions. The aim is rather to indicate, for the sake of the discussions that follows, that there is at least a reasonable possibility that consciousness has a genuine causal role, and that this connects strongly with the notion of information. For a more detailed discussion the reader is advised to consult the references given below, as well as in Van Gulick (2014: 35-42) and Revonsuo (2006). Let us now consider a number of suggestions about the causal role of consciousness.

It is common to claim that conscious mental processes provide a flexible and adaptive type of control, as opposed to unconscious automatic processes (Anderson 1983). Even if these latter can be quick, they are also relatively fixed and pre-determined, and thus not particularly effective in unexpected situations (Penfield 1975). Also, when the challenge is to learn new skills, conscious attention is typically assumed to be important at the early stages of learning (Shiffrin and Schneider 1977).

It has been suggested that organisms that are conscious of their own and others' mental states have a better ability to interact, cooperate and communicate. The idea is that such meta-mental or "higher-order" consciousness would enable a better capacity for social coordination, which in turn can be thought to provide adaptive advantage (Humphreys 1982; Van Gulick 2014: 38).

It has further been suggested that conscious experiences enable a more unified and integrated representation of reality, which allows for a more flexible response in various situations (Campbell 1994; Van Gulick 2014: 38-9; Tononi and Koch 2014).

It is a well-known suggestion that information in conscious mental states is globally available to a number of different mental subsystems or "modules", and can thus be made use of in many different ways in behavior (Baars 1988). In contrast, it is argued that non-conscious information is usually available only to special mental modules and has a more limited effect upon behavior and action (Fodor 1983). (However, Rosenthal (2009) thinks it is unclear that a state's potential to have global effects coincides with its being conscious.)

When it comes to free will, it seems that conscious experience not only presents us with the options to choose from (at least sometimes), it also seems to be a prerequisite for such freedom. Mustn't one be conscious to be able to make a free choice at all (Van Gulick 2014: 41)? One should note that 
researchers such as Velmans have suggested that there can be unconscious free will; but it is not obvious that a decision made unconsciously can be considered truly free.

Finally, it has been suggested that certain conscious states, such as pleasure and pain have an intrinsic motivating force (e.g. attraction), as an indivisible part of the experience itself. The idea is that such a force cannot be reduced to non-conscious properties (for a brief account of the various viewpoints on this issue, see Van Gulick 2014: 41-2).

Antti Revonsuo (2006) has considered the causal powers of consciousness (or the "phenomenal level" as he calls it) in the light of various studies on blindsight, implicit perception, non-conscious visually guided actions, and similar phenomena. He acknowledges that there are complex information processing mechanisms in the brain that in themselves are non-conscious, or in his terms, "realize no phenomenal level of organization". However he emphasizes that such non-conscious "zombie systems" seem to have only limited causal powers in guiding organism-environment interaction, whereas the contribution of consciousness (or the "phenomenal level") seems to be decisive for meaningful interactions with our environment.

He further considers disorders, such as epileptic automatisms and sleepwalking, which seem to turn the whole person into a non-conscious zombie, and notes that a careful examination of such zombies reveals that non-conscious organism-environment interaction, while complex, is typically pointless. He concludes (2006: xxiii-xxiv):

"... other types of disorders show that the simulated phenomenal world in the brain has unique causal powers in determining the behavioral trajectories of our physical bodies. In the light of the evidence from these disorders, consciousness surfaces as a causally potent biological system with unique causal powers. Therefore, we need not worry about epiphenomenalism any longer."

We note here that Revonsuo's reference to the way in which the simulated phenomenal world in the brain determines behavioral trajectories of bodies is interestingly analogous to Bohm's notion that active information encoded in the quantum field determines the trajectories of particles at the quantum level (we will discuss this latter idea in section 5). We also note that to truly avoid epiphenomenalism or reductionism, Revonsuo needs to show how conscious experiences qua conscious could possibly play a genuine causal role in guiding the physical organism, without violating the laws of physics (or the causal closure of the physical domain). This is of course connected to the problem of mental causation, a solution to which we are trying to sketch in this paper.

\section{How the causal efficacy of consciousness connects with information}


Let us now see how the above suggestions make a link between consciousness and information. We can understand "more flexible control" as flexibility in the way that information can be used to guide the organism. It seems that consciousness makes possible such flexibility. Unconscious information just "acts" when it is activated, according to an automatic routine. If there are items of unconscious information that imply mutually exclusive actions, then presumably the "stronger" information wins, and this may take place without conscious experience ("stronger" here may be assumed to correspond to e.g. a higher level of neural activity). However, it seems possible that when a person is conscious of an item of information, at least some (automatic) activity of that information can be suspended. Also, it seems obvious that at least in some situations a person can review a number of different options, and choose the one that seems best in the given situation. (In this way consciousness, flexible control and free will seem related). Of course, which option is in the end chosen may not be the result of a completely "free" choice, but is instead determined by some further information which arises when reviewing the options, with a content like "it is reasonable to do X" (cf. Bohm 1990).

We also noted that it has been suggested that organisms that are conscious of their own and others' mental states have a better ability to interact, cooperate and communicate. "Conscious of" can here be understood to include "having meta-level information about". This connects with higher order theories of consciousness which assume that what makes a given mental state conscious is that there exists a higher level of (typically) unconscious mental state, which has the content that one is in the first order mental state or activity (Rosenthal 1997). Thus, consciousness is not assumed to be a neural or computational property, but rather something that arises when initially non-conscious mental states are related in a suitable way. It seems quite natural to think about such meta-mentality in terms of information. We could say that meta-mentality involves higher-order "information about information" rather than just first-order "information about the environment". In these terms, higher-order theories of consciousness suggest that consciousness essentially involves information about information. A simple possibility would be to postulate that what makes a given informational state conscious is that there exists a higher level of (typically) unconscious information, which has the content that one is in the first order informational state. When it comes to the causal efficacy of consciousness, the question is whether having meta-level information (and consciousness) in this sense implies a better ability to interact, cooperate and communicate. In section 8 we will briefly note how in the Bohmian scheme active information at a given level can organize the behavior of elements at a lower level. The challenge here, too, is to find out whether being conscious of active information gives the organism some special advantages, when it comes to interaction, cooperation and communication. 
We further mentioned the suggestion that conscious experiences enable a more unified and integrated representation of reality, which allows for a more flexible response in various situations. To understand this feature better, we can usefully quote van Gulick (2014: 38-9):

Conscious experience presents us with a world of objects independently existing in space and time. Those objects are typically present to us in a multi-modal fashion that involves the integration of information from various sensory channels as well as from background knowledge and memory. Conscious experience presents us not with isolated properties or features but with objects and events situated in an ongoing independent world, and it does so by embodying in its experiential organization and dynamics the dense network of relations and interconnections that collectively constitute the meaningful structure of a world of objects.

This reminds us about the fact that the information we meet in consciousness is highly integrated and structured and also meaningful in various ways. Van Gulick acknowledges that non-experiental sensory information can also have an adaptive effect on behavior (e.g. as seen in reflexes). However, he draws attention to the work of Lorentz (1977) and Gallistel (1990), which suggest that conscious experience provides a more integrated representation of reality, which in turn enables more flexible responses. If we consider this feature in informational terms, it seems that certain kind of information only becomes available and, especially, flexibly usable to the organism in conscious experience. This connects with the previously mentioned issues of flexible control and free will, in the sense that consciousness, flexible control, free will and unified and integrated representations are all interconnected. Unified and integrated representations, especially when consciously experienced, provide the "free will" rich information about the available options and this enables flexibility in the control of the organism.

There are a number of other researchers who emphasize that consciousness involves an integrated representation in the form of a "virtual reality" or "world-simulation". Revonsuo, for example, characterizes conscious experience in dreams as a complex, organized, temporally progressing worldsimulation. During waking we also experience subjectively an internal, phenomenal, simulated world, which we take to be the "real" world, when consciousness happens to be online with the external physical world (Revonsuo 2015: 65). And as we already saw above, for Revonsuo the simulated phenomenal world in the brain is causally efficacious in that it determines the behavioral trajectories of our physical bodies. Here we can ask what is the nature of a world-simulation. It seems natural to think it as some kind of structure of information that is meaningful and has phenomenal properties. And given that this world-simulation guides the organism, it is natural to think of it as a kind of active information in the Bohmian sense that will be explained later.

Let us then move on to consider the suggestion that information in conscious mental states is globally available to a number of different mental subsystems or "modules", and can thus be made use of in many different ways in behavior. This feature, together with the issues discussed previously, helps 
to explain the flexible control that consciousness seems to enable. We saw above that information in conscious experience is typically very rich in its content - unified and integrated. If consciousness further means that such information becomes globally available to many different subsystems, it clearly becomes easier to understand why consciousness enables more flexible control. To put it briefly, the idea is that consciousness both enables the sort of information that flexible control requires, and it also makes it possible for such information to reach the subsystems that are required in the execution of the control.

In recent years much attention has been given to Tononi's Integrated Information Theory of Consciousness (Tononi and Koch 2014; Oizumi et al. 2014). There are various reasons why Tononi thinks the concept of information is needed in a theory of consciousness. To account for the fact that consciousness is differentiated (i.e. that each experience has a specific set of phenomenological distinctions), a system of mechanisms must specify a differentiated conceptual structure via a process of in-forming (we will see later that Bohm's notion of active information likewise refers to a process of informing, though in a somewhat different sense). Tononi further says that to account for the irreducible unity of consciousness (i.e. that each experience is irreducible to non-interdependent components), there has to be integrated information, in the sense that the conceptual structure specified by the system is irreducible to that specified by non-interdependent sub-systems. More technically, the presence of integration (characterized by big phi or $\Phi$ ) means that a partitioning of a system of mechanisms would destroy several cause-effect repertoires and change others.

Tononi's theory tries to explain what consciousness is in terms of the notion of information. But the theory also suggests that consciousness as integrated information makes a difference to the behavior of the organism. Tononi and Koch (2014) write: “...a brain having a high capacity for information integration will better match an environment with a complex causal structure varying across multiple time scales, than a network made of many modules that are informationally encapsulated." And given the hypothesis that consciousness is integrated information, this implies that consciousness enables a better match with the environment and consequently more adaptive behaviour.

We have already briefly considered the relation of free will and consciousness above, and will return to this issue in section 8. Van Gulick's review also drew attention to the suggestion that certain conscious states, such as pleasure and pain have an intrinsic motivating force (e.g. attraction), as an indivisible part of the experience itself. The idea is that such force cannot be reduced to non-conscious properties. This suggests that consciousness not only enables information to be integrated and globally available, but that it also involves (perhaps gives rise) to "forces", such as attraction. Again, we will return to consider this interesting suggestion when discussing the notion of active information in section 8. 
Van Gulick's review (as well as Revonsuo's and Tononi's views) make a reasonably strong case for the idea that consciousness has genuine causal powers. Now, presumably each particular argument for such causal efficacy is subject to potentially serious criticisms, but I think that it is fair to say that together they imply that the question is at least an open one. It at least seems to make a difference to the behavior of an organism whether or not it is conscious. I also drew attention to the way many of the suggestions about the causal efficacy of consciousness involve a link between consciousness and information. In the rest of the paper I will try to understand this link better by discussing it in the context of a new notion of active information that is extended all the way into physics. However, before doing that I want to briefly meet another challenge. For as was already hinted above, contemporary philosophers of mind often suggest that consciousness cannot have genuinely causal powers if we stay within the physicalist scientific world picture. We need to address this issue briefly before proceeding.

\section{Philosophy of mind: consciousness has no causal powers?}

Much of contemporary Anglo-American analytical philosophy is committed to physicalism, which means that philosophers assume that everything is physical, or everything is in an appropriate way dependent (or "supervenient") upon the physical. However, many philosophers find it difficult to simply reduce the mental to the physical, and they thus defend a doctrine known as "non-reductive physicalism". This typically holds that mental properties are non-physical properties that, however, depend or supervene upon the physical. Note that "mental" here is not taken to be synonymous to "conscious", but includes even such possibly non-conscious properties as intentionality (in the sense of the "directedness or "aboutness" of mental states).

The trouble with non-reductive physicalism is that it seems to leave the mental causally inefficacious or epiphenomenal. If the mental is non-physical, it seems impossible to understand how the mental could be the cause of physical effects. Even the notion of mental-physical dependence or supervenience doesn't seem to help here. Some philosophers (e.g. Yablo, Lewis and Kim) have developed some ingenious ways to make the idea of genuine mental causation plausible (see Ritchie 2008). However, it seems that even these fail to tell us how mental properties (conceived as nonphysical) could possibly influence the physical course of events. There thus seems to be no genuine causal role for mental properties in contemporary non-reductive physicalism. This is a very unsatisfactory situation. However, to go back to, say, interactive substance dualism seems equally unsatisfactory. Thomas Nagel (2005) has succinctly summarized the situation: "Neither dualism nor materialism seems likely to be true, but it is not clear what the alternatives are". 
Note that this apparent epiphenomenalism of the mental is particularly troublesome for our above discussion about the causal role of conscious experience. It is not at all obvious that conscious experiences are physical or material in any traditional sense (remember e.g. David Chalmers's (1996) discussion of the 'hard problem' of consciousness). Thus contemporary non-reductive physicalism seems forced to declare consciousness to be an epiphenomenon. Reductive physicalism resolves the issue trivially by assuming that conscious experiences are physical states. But for those who do not understand how conscious experience could possibly be a physical state, this 'resolution' is not of much value.

We have noted that non-reductive physicalism implies that consciousness is epiphenomenal, but how seriously should we take the non-reductive physicalists's arguments? For if one examines the views of many of the leading physicalists (whether reductive or non-reductive), one is struck by the fact that hardly any attention is given to what seems to be the most fundamental of the natural sciences, namely (fundamental) physics. This seems to be in violation of the very principles the physicalists have usually set to themselves, namely that they ought to base their metaphysics upon the best theories in the natural sciences. A particularly sharp criticism of such tendencies in philosophy has recently been made by Ladyman and Ross 2007. They write, for example, that “...standard analytic metaphysics (or 'neoscholastic' metaphysics as we call it) contributes nothing to human knowledge and, where it has any impact at all, systematically misrepresents the relative significance of what we do know on the basis of science" 2007. Such 'neo-scholastic' metaphysics also includes analytic philosophy of mind, in so for as this gives little attention to the results of modern science, including fundamental physics. Ladyman and Ross's view is extreme, but I think they are correct in drawing attention to certain weak points in contemporary philosophy of mind. If we want to claim that the physical world leaves no room for the causal powers of consciousness, we should justify our view on the basis of best theories in physics. And as we will see in the next section, it is not clear that, say, quantum theory excludes in principle the causal powers of consciousness. On the contrary, a natural extension of quantum theory might well make room for mental properties and even conscious experience in our scientific world picture.

\section{Information in the ontological interpretation of quantum theory}

Can quantum theory throw any new light upon the nature of information, which might also help us to understand the relationship between consciousness and information, and the causal powers of consciousness? I suggest that the best place to start exploring this issue is David Bohm's interpretation of quantum theory, in its later form developed in co-operation with Basil Hiley (Bohm and Hiley 1987, 1993). 
To understand the significance of Bohm's work for the mind-matter problem it is necessary to understand the development of physics in the $20^{\text {th }}$ century. When quantum theory was emerging, physicists were trying to make sense of puzzling features such as wave-particle duality and a little later, entanglement. In particular they were attempting to develop ontological models of quantum systems such as electrons. In the 1920s Louis de Broglie came up with the idea of an electron being a particle guided by a pilot wave, while Schrödinger was trying to describe the electron as some kind of a physical field. These models had some difficulties, though in retrospect we can see that at least de Broglie's ideas could have been developed further (Bacciagaluppi and Valentini 2009). What happened however was that the so-called "Copenhagen interpretation" won the day in the 1920s. There are actually many different versions of this interpretation, but it is typical of them that they emphasize epistemology - in the sense our ability to predict the statistical results of measurement, rather than ontology - in the sense of a model of what quantum reality may be like, also when we are not making measurements. As a result, physicists were not able to offer a new notion of objective physical reality, which philosophers could then use when discussing ontological issues, such as the mind-matter relation.

It is here that Bohm comes in. In the early 1950s, after discussions with Einstein in Princeton, he independently rediscovered de Broglie's theory and formulated it in a more coherent way, providing a first consistent realistic model of quantum systems (Bohm 1952). Bohm's interpretation was initially resisted, but is today more and more widely acknowledged as one of the key possible interpretations of quantum theory. Later on further ontological models were proposed, for example Everett's 1957 “many worlds" interpretation and Ghirardi, Rimini and Weber's 1986 objective collapse theory, and currently the nature of quantum reality is intensively debated within the philosophy of physics community (see e.g. the anthology The Wave Function: Essays on the Metaphysics of Quantum Mechanics, edited by Alyssa Ney and David Albert). We do not know which ontological interpretation (if any) is correct, but each may reveal something significant about the nature of physical reality at a very fundamental level. One should note that there are by now also different versions of the Bohm theory. Much attention has in recent years been given to a minimalist version known as "Bohmian mechanics" (see e.g. Goldstein 2013). Bohm himself developed since the mid-1970s, with Basil Hiley, a philosophically more radical version they called the "ontological interpretation", culminating in their 1993 book The Undivided Universe.

How, then, might Bohm's theory be relevant to the mind-matter relation and to the causal status of consciousness in particular? It postulates that an electron is a particle, always accompanied by a new type of field, which guides its behavior - thus the name "pilot wave theory" which is sometimes used. Jack Sarfatti has characterized the Bohmian electron imaginatively by saying that it consists of a "thought-like" pilot wave, guiding a "rock-like" particle. This metaphor suggests that matter at the 
quantum level is fundamentally different from the sort of mechanical matter of classical physics that is presupposed in philosophy of mind by typical materialists. If even the basic elements that constitute us have "thought-like" and "rock-like" aspects, then it is perhaps not so surprising that a very complex aggregate of such elements (such as a human being) has a body, accompanied by a mind that guides it.

But, one might think, this is merely a vague metaphor. Now, Bohm himself realized in the early 1980s that the pilot wave might be more literally "thought-like" in a very interesting sense. He considered the mathematical expression of the so-called quantum potential, which describes the way the pilot wave affects the particle. He realized that the quantum potential, and thus the effect of the wave upon the particle, only depends on the form or shape of the wave, not on the size or amplitude of the wave (mathematically, the quantum potential depends only on the second spatial derivative of the amplitude of the wave). He went on to suggest that the quantum wave is literally putting form into, or in-forming the motion of the particle along its trajectory, rather than pushing and pulling it mechanically.

Note that we are here talking about information for the electron, not information for us - we are thus thinking about information as an objective commodity that exists out there in the world, independently of us, guiding and organizing physical processes. The form of the quantum wave reflects the form of the environment of the particle - for example the presence of slits in the famous two-slit experiment. In this experiment, electrons arrive one by one at the detecting screen at localized points, suggesting that they are particles. Yet as we keep on watching, the individual spots build up an interference pattern, suggesting that each individual electron also has wave properties. Remember that in the Bohm theory the electron is seen as a particle and a wave. In the two-slit experiment the particle goes through one of the slits. The wave goes through both slits, interferes and guides or in-forms the particle in such a way that an interference pattern is formed, as many electrons pass through the slit system. It thus seems that with the help of the notion of active information we can have a realist interpretation of the quantum theory, without the usual puzzles, such as Schrödinger's cats, many worlds or the consciousness of the observer producing physical reality (for details see Bohm and Hiley 1987, 1993).

What happens with the electron is somewhat analogous to a ship on autopilot, guided by radar waves that carry information about the environment of the ship. The radar waves are not pushing and pulling the ship, but rather in-forming the much greater energy of the ship. Bohm generalized this into a notion of "active information" - which applies in situations where a form with small energy enters and informs a larger energy. We see this not only with various artificial devices, but also in the way the form of the DNA molecule informs biological processes, and even in the way forms act in human subjective experience (for example, seeing the form of a shadow in a dark night and interpreting it as "danger" may 
give rise to a powerful psycho-somatic reaction). Indeed, Bohm (1990) sketched how the active information approach could be developed into a theory of mind and matter.

While the radar-wave analogy helps to understand the Bohmian electron, it is important to realize that the quantum potential has some radically holistic properties that go beyond what is implied by such mechanical analogies. In particular, in the many-body system there can be a non-local connection between particles that depends on the quantum state of the whole, in a way that cannot be expressed in terms of the relationships of the particles alone. Bearing in mind that this quantum state involves active information, we can note an interesting connection to Tononi's idea of integrated information. It is likely that the many-body quantum state involves the most radically holistic (integrated) information that science has thus far detected, thus making it interesting to consider its role when trying to understand consciousness as integrated information.

\section{Bohm's sketch for a theory of the relation of mind and matter}

Bohm proposed that we understand mental states as involving a hierarchy of levels of active information. We typically not merely think about objects in the external world, but we can also become aware of our thinking. He suggested that such meta-level awareness typically involves a higher level of thought. This higher level gathers information about the lower level. But because its essential nature is active information, it not merely makes a passive representation of the lower level. Rather, the higher level also acts to organize the lower level, a bit analogously to the way the active information in the pilot wave acts to organize the movement of the particle. (In particular, the higher level of thought can organize the content in the lower level into a coherent whole. This could be seen as a kind of "integrated information" and suggests yet another connection with Tononi's Integrated Information Theory of consciousness.) And of course, we can become aware of this higher level of thought from a yet higher level, and so on.

How does then mind, understood as a hierarchy of levels of active information, connect with matter in the Bohmian scheme? First of all, he suggested that it is natural to extend the quantum ontology. So just as there is a pilot wave that guides the particle, there can be a super-pilot wave that guides the $1^{\text {st }}$ order pilot wave, and so on. (He claimed that such an extension is "natural" from the mathematical point of view.) Now it seems that we have two hierarchies, one for mind and another for matter. His next step was to postulate that these are the same hierarchy, so that there is only one hierarchy. This then allows, at least in principle, for a new way of understanding how mind can affect the body. Information at a given level of active information in the mind can act downwards, all the way to the active information 
in the pilot waves of particles in, say, the synapses or neural microtubules, and this influence can then be amplified to signals in motor cortex, leading to a physical movement of the body.

Bohm's proposal differs strongly from the usual theories in cognitive neuroscience. Most neuroscientists ignore quantum considerations, and seek the "neural correlates of consciousness" in some macroscopic neural phenomena, which can presumably be understood in terms of classical physics. Yet Bohm is proposing that mind, understood as a hierarchy of levels of active information, is implemented in (or perhaps even identical with) a hierarchy of super-quantum fields. However, these fields are not separate from the macroscopic neural processes. On the contrary the role of the fields is in the end to gather information about the manifest neural processes, and on the basis of what this information means, to organize and guide them.

One should acknowledge that it is a tremendous challenge to work out an empirically testable theory along the Bohmian lines. The ideas described above provide a scheme for such an endeavor, rather than a fully developed theory. Bohm and Pylkkänen (1992) were discussing ways to develop the scheme in the late 1980s and early 1990s. In a later development, Hiley and Pylkkänen (2005) discussed the prospects of applying the Bohm scheme to Beck and Eccles's quantum model of synaptic exocytosis. While this may be a small step forward, problems remain. For example, Henry Stapp has pointed out that the sort of interference of the mind upon the laws of quantum mechanics that the Bohmian scheme involves can lead to serious problems with special relativity (private communication). This is a challenge that future research along Bohmian lines needs to face.

While the possibility of non-negligible quantum effects in the brain is often dismissed as implausible, there are interesting recent advances in quantum biology. And it is already part of mainstream neuroscience that the retina acts to amplify the effects of individual photons. Also, researchers such as Roger Penrose and Stuart Hameroff have discussed in great detail how quantum effects might play a role in neural processes via quantum coherence and collapse in neural microtubules. Connecting the Hameroff-Penrose work with the Bohm scheme is one potentially fruitful line for future research. Indeed the author has began to explore these connections together with Hameroff and the philosopher Rocco Gennaro, who is a specialist on higher-order (HO) theories of consciousness (which seem to fit together with Bohm's idea of the mind as a hierarchy of levels of information). (For an early result of this co-operation, focusing on combining $\mathrm{HO}$ theories with Penrose and Hameroff's ORCHOR hypothesis, leading to "deeper order thought" (DOT) see Hameroff et al. (2014).

Note that Bohm introduced a new category, namely information to the debate. Is information physical or mental? He suggested that it is simultaneously both physical and mental, or has these two as its aspects. This sort of view is called a double-aspect theory in philosophy of mind. The traditional worry with double-aspect views is that it is left into a mystery what the underlying thing, which has the 
aspects, is. The hypothesis that information is the fundamental, underlying feature of reality can be seen as a way to alleviate this worry.

\section{Understanding consciousness in the active information scheme}

A common criticism of contemporary theories in the philosophy of mind - such as identity theory and functionalism - is that they leave out conscious experience, instead of explaining it (Searle 1992). How might conscious experience fit into the active information scheme? In particular, is it possible to understand the causal status of consciousness in this scheme? While Bohm saw nature as a dynamic process where information and meaning play a key dynamic role, he assumed that " 99.99 per cent" of our meanings are not conscious (Bohm in discussion with Renée Weber (Weber 1987: 439)). Thus, for example, he thought it obvious that the particles of physics are not conscious. But how can one then address the problem consciousness in this scheme? In other words, why is there sometimes conscious experience associated with the activity of information (as seems obvious at least with humans and higher animals)? Why doesn't all the activity of information in humans proceed "in the dark", as it seems to do in physical and biological processes in general? And does the presence of consciousness make a causal difference? Bohm himself did not say much about the hard problem of consciousness (he died a little before the hard problem was made the center of attention by David Chalmers in the 1994 Tucson consciousness conference). However, the author has suggested that the most natural context to explore this issue is some version of a higher order (HO) theory of consciousness (Pylkkänen 2007: 247). Let us here expand somewhat on this idea.

As we saw above, the basic idea of higher-order theories of consciousness, when expressed in terms of the notion of information, is to postulate that what makes a given mental state (or level of information or mental activity) conscious is that there exists a higher level of (typically) unconscious information, which has the content that one is in the first order mental state or activity.

Note also that David Chalmers famously suggested that we tackle the hard problem of consciousness with a double-aspect theory of information. The idea is that information is a fundamental feature of the world, which always has both a phenomenal and a physical aspect. Now, we could take this idea to the Bohm scheme and postulate that active information, too, has phenomenal properties. This then raises the question about what we should think about the active information in the pilot wave of an electron. Does it, too, have phenomenal properties in some sense? Bohm went as far as to say that electrons have a "primitive mind-like quality", but by "mind" he was here referring to the "activity of form", rather than conscious phenomenal experience in any full sense. 
I think that it is reasonable to combine Chalmers's hypothesis to active information, but we need to restrict the hypothesis. For example, we could say that certain kind of active information (for example, a holistic active information that is analogous to quantum active information) has the potentiality for phenomenal properties, but this potentiality is actualized only in suitable circumstances (for example, when a given level of active information is the intentional target of a higher level of active information; or if we want to follow an approach similar to that of Tononi, we could say that suitably integrated active information is conscious). Of course, this also opens up the possibility for genuine artificial consciousness. If we could implement quantum-like holistic active information in an artificial system and set of up a suitable higher-order relationship of levels in the system, phenomenal properties should actualize themselves, according to this hypothesis. (Or, in a Tononian approach, if active information is suitably integrated in an artificial context, it would be conscious.)

We should acknowledge that Bohm and Hiley's proposal about active information at the quantum level is radical and somewhat controversial, for they are in effect suggesting that this type of information ought to be acknowledged as a fundamental - perhaps THE fundamental - category of physics. Indeed, they wrote in 1984: "The notion of a particle responding actively to information in the [quantum] field is ... far more subtle and dynamic than any others that have hitherto been supposed to be fundamental in physics". This proposal is still mostly ignored within the physics community. There are some technical issues with the proposal, but in my view a major reason is that it goes so much against the prevalent mechanistic way of thinking in physics. However, some leading thinkers do take it seriously, for example Quentin Smith (2002). Also, an interesting adaptation of the active information scheme to neuroscience has been proposed by Thomas Filk (2012). In the field of the social sciences, Andrei Khrennikov (2004) has made imaginative use of the proposal and the Bohm theory has also been applied to financial processes by Olga Choustova (2007) and Emmanuel Haven (2005). Of course, the notion of "quantum information" has been widely discussed in recent years (see. e.g. Bouwmeester et al. 2000). The advantages of the concept of active information over quantum information, when discussing some quantum experiments, have been argued for by Owen Maroney (2002); see also Maroney and Hiley (1999).

To summarize: Bohm's suggestion was that a natural extension of his ontological interpretation of the quantum theory can include mental processes and even conscious experience into a single coherent view. From the point of view of the question about the causal powers of consciousness this view is particularly promising, for it makes it - at least in principle - possible to understand how conscious experience, via its effects upon information, could make a difference to physical process. If we can provide an intelligible theory about how conscious experience can make a difference to information, this scheme provides a view of how such informational differences can then affect manifest physical 
processes (see also Hiley and Pylkkänen 2005). We have hinted that this question can be approached within some of the already existing available theories of consciousness - for example higher order theories or Tononi's Integrated Information Theory.

\section{Active information and the causal powers of consciousness}

The view described above sketches how information content might affect manifest physical processes (e.g. bodily behavior) in a way that is coherent with the principles of physics. We also already touched on the question about causal role of consciousness in the active information scheme. Let us now consider this role in more detail, by returning to the suggestions about the causal role of consciousness mentioned in Section 2. First of all, how can we understand the idea that consciousness enables more flexible control in the context of the active information view? More flexible control means, for example, that the organism is able choose from different options the one that best fits the situation, instead of having to mechanically follow one of the options. In Bohmian terms this means that consciousness enables the organism to suspend the activity of information. The way this works is that one is aware of information that means something like "it is reasonable to consider different options before acting". And when one finally acts, this is based on information that means "it is reasonable to do X". In other words, flexible control in the Bohmian view seems to involve higher-order, meta-level information that we are conscious of (while typically, according to higher order theories, we need not be conscious of the higher-order thought itself).

When it comes to better social coordination, Bohm's view involves a notion he calls "common pools of information" (Bohm 1990). This notion applies strikingly well at the quantum level (e.g. in the Bohmian view of superconductivity) where the behavior of a system of particles can in some situations be organized by information in the so called many-body wave function. The particles act together in an organized way (e.g. electrons may pass obstacles in a wire, which results in very low resistance). Information at the level of human cognition operates presumably according to different principles that information at quantum level. However, when a group of people communicate with each other (e.g. in a group discussion) they begin to build up a common pool of information. This enables the group develop common intentions and carry out common actions. Suppose, for example, that a group of 8 people needs to carry a very heavy grand piano upstairs along a narrow staircase. They need to exchange information and make sure that they each understand what they are supposed to do. Again, it is hard to imagine that such joint tasks requiring collective coordination could take place without some consciousness of the shared information. However, it is an experimental question to what extent such collective action is possible without conscious awareness. Consider, for example, a situation where the 
group needs to carry the piano through a very narrow opening. For a radical approach to the shared intentionality of the mother-infant relationship, making use of quantum principles in a phenomenological context, see Flender et al. (2009).

We also considered the suggestion that consciousness enables more unified and integrated representation. The tricky question here is whether the information first gets unified and integrated in pre-conscious processes, and is then presented to consciousness. Or does consciousness play a role in the very unification and integration of the information (Van Gulick seems to favour the latter alternative)? I am inclined to think that much of the unification and integration of information takes place (largely) without consciousness, but that consciousness is needed for such information to be flexibly usable in the control of behaviour (of course, in the Tononian approach one would say that sufficiently integrated information constitutes consciousness). In the Bohmian picture it is assumed that typically such information tends to act, even if it is not consciously attended to. Conscious attention may then make the response of information stronger, or lead to the suspension of action and reflection of the different options.

The idea that consciousness involves more global access can also be naturally understood in terms of the notion of active information. If information is consciously attended to, this may start what Bohm calls a "signa-somatic" flow: the significance of the information acts somatically toward a more manifest level in the brain. Global access means that the significance can affect many different modules.

When it comes to free will, Bohm used to emphasize that true freedom is typically limited by our lack of knowledge - both about the consequences of one's actions and about our true motives. He refers to Schopenhauer when he writes: “... though we may perhaps be free to choose as we will, we are not free to will the content of the will. ... Is there any meaning to freedom of will when the content of this will is ... determined by false knowledge of what is possible..." (Bohm 1986). In a more positive vein, he writes:

\footnotetext{
"How, then, is it possible for there to be the self-awareness that is required for true freedom? ... I propose that self-awareness requires that consciousness sink into its implicate (and now mainly unconscious) order. It may then be possible to be directly aware, in the present, of the actual activity of past knowledge, and especially of that knowledge which is ... false. ... Then the mind may be free of its bondage to the active confusion that is enfolded in its past" (Bohm 1986).
}

By "the implicate order" Bohm above refers (roughly) to the more subtle levels of active information which include long-term memory and from which the part of the content of conscious experience unfolds. It is clear that for Bohm free will requires consciousness. However, it is not enough that we are 
conscious of the options that we typically face in a situation when we are about to make a choice. We also need to be aware - and thus free - from falsity in the past knowledge that we typically unconsciously hold and on the basis of which we tend to react and make our choices.

Let us finally consider intrinsic motivation in the light of the Bohmian view. What is interesting here is that Bohm emphasizes that information is typically active (while passive information is a special case). One possibility is that the presence of consciousness increases the level of activity of the information. Thus, for example, consciousness of information with an attractive content may be needed to awaken desire or make that desire more intense. At the same time conscious awareness of the negative consequences of carrying out a particular desire may lead to the suspension of action. In Bohmian terms, all these phases involve active information. For example, desire informs us to carry out a certain action $\mathrm{X}$, while information about the consequences of the action may result in information with the content "it is not reasonable to do X".

\section{Concluding discussion}

We have taken some help from fundamental physics to support the idea that conscious experiences can, at least in principle, be causally efficacious in a physical world, contrary to what much of contemporary physicalism suggests. Yet we have admittedly only scratched the surface of this difficult topic. Basically, I have assumed that consciousness (understood as something that arises due to higher order information and/or information integration) can influence lower-level information, and information in turn can influence physical processes "signa-somatically", as Bohm would put it.

The Bohmian view we considered suggests that nature can be understood as a two-way movement between the aspects of soma (the physical) and significance (information, meaning, the mental). Consciousness comes in here, but only at the higher, subtler levels, where, say, suitable higherorder relations (and/or a sufficient degree of information integration) prevail, depending upon which theory of consciousness we are relying upon. Thus the active information view is consistent with the idea - also supported by recent experimental work - that much of our most sophisticated brain functions work totally independent from consciousness. Yet the active information view also makes room for the genuine causal powers of consciousness, and in this way can accommodate such causal efficacy of consciousness as is suggested by Van Gulick, Revonsuo and others. Bohm himself did not address very explicitly the causal powers of consciousness, but I think it is reasonable to assume that his scheme makes such powers in principle possible. To fully explain that scheme is, however, not possible here, and the interested reader is referred to a more detailed study (Pylkkänen 2007). 
One important potential criticism of the active information approach has to do with the notion of information that is presupposed. Is it really justified to use the term "information" to describe the sorts of processes connected to the quantum field? One could examine this question in the light of the recent developments in the philosophy of information (e.g. Floridi 2015). Floridi distinguishes between environmental and semantic information; and semantic information can be further distinguished into factual and instructional information. The quantum active information is about something (the environment, slits, etc.), it is for the particle and it helps to bring about something (a certain movement of the particle). This suggests that it is semantic and has both factual and instructional aspects, though this issue needs to be explored more carefully in future research. Also, Maleeh and Amani (2012) have usefully considered active information in relation to Roederer's (2005) notion of pragmatic information, suggesting that only biological systems are capable of "genuine" information processing. I think one can argue that Bohmian quantum information potential involves genuine information processing (indeed, the most fundamental kind of genuine information processing science has thus far discovered), but this will also be explored in future research.

I would like to end by reflecting upon the quote from Plato's Phaedo that was given at the outset. Plato there thinks it obvious that our physical actions depend upon "the choice of the best", while a typical materialist would say that insofar as physical actions are determined, they are determined by the physical state in a previous moment (including "bones and muscles"). Now, I think that the active information view allows for a naturalistic grounding of Plato's view. In their 1984 article Bohm and Hiley note that there are good reasons for expecting that quantum theory, and therefore the notion of a quantum information potential, would be relevant when we are studying consciousness itself, as based on the material structure of the brain and nervous system:

“...it may well be that in our mental processes, the quantum information potential is significant (as is, for example, suggested by the fact that information regarded as correct is active in determining our behaviour, while as soon as it is regarded as incorrect, it ceases to be active). The quantum theory may then play a key part in understanding this domain.” (1984: 269)

The above implies that our veridicality judgements play a key role in determining whether or not information acts. For example, if I judge a shadow in a dark night to mean "an assailant" and thus "danger", this typically gives rise to a powerful psychosomatic reaction; if I a little later notice that it was merely a shadow of a branch (i.e. that the earlier judgement was incorrect), I will typically calm down. We could expand the idea toward Plato by assuming that our ethical judgements (e.g. "the choice of the best") can typically also affect the way information is activated, and consequently our behaviour. 
The quantum theoretical active information scheme enables such activity of information to reach all the way to the level of fundamental physics, and this way we can begin, in a new way, to make sense of a perennial puzzle in Western philosophy, namely the place and role of minds, meanings and morals in the physical world.

\section{References}

Anderson, J., The Architecture of Cognition. Cambridge, MA: Harvard University Press, 1983.

Baars, B., A Cognitive Theory of Consciousness. Cambridge: Cambridge University Press, 1988.

Bohm, D., A Suggested Interpretation of the Quantum The- ory in Terms of "Hidden Variables" I and II, Phys. Rev., 85 (2): 166-179 and 180-193, 1952.

Bohm, D. Time, the implicate order and prespace. In Physics and the Ultimate Significance of Time. Bohm, Prigogine and Process Philosophy; D. Griffin Ed. State University of New York Press: New York, 1986, pp. 177-208.

Bohm, D. A new theory of the relationship of mind and matter. Philosophical Psychology 1990, 3, 271286.

Bohm, D. Soma-significance and the Activity of Meaning. In The Essential David Bohm; L. Nichol Ed., Routledge: London, 2003; pp. 158-182.

Bohm, D. and Hiley, B.J., Measurement understood through the quantum potential approach. Foundations of Physics 1984, 14, 3, 255-274.

Bohm, D. and Hiley, B. J., An Ontological Basis for Quantum Theory: I. Non-relativistic Particle Systems. Phys. Rep. 144 (6): 323-348, 1987.

Bohm, D. and Hiley, B.J. The Undivided Universe. An Ontological Interpretation of Quantum Theory. Routledge: London,1993.

Bohm, D. and Pylkkänen, P., Cognition as a movement toward coherence. Unpublished manuscript, 1992. 
Bouwmeester, D., Ekert, A. and Zeilinger, A.K. Eds., The Physics of Quantum Information: Quantum Cryptography, Quantum Teleportation, Quantum Computation. Heidelberg and Berlin: Spinger, 2000.

Campbell, J., Past, Space, and Self. Cambridge, MA: MIT Press, 1994

Chalmers, D. The Conscious Mind. Oxford University Press: Oxford, 1996.

Choustova, O. Toward quantum-like modeling of financial processes, J. Phys.: Conf. Ser. 70, 012006, 2007.

Filk, T. Quantum-Like Behavior of Classical Systems, in Quantum Interaction: Lecture Notes in Computer Science Vol. 7620, 2012, pp. 196-206.

Flender, C., Kitto, K., and Bruza, P., Non-separability of shared intentionality. In P. Bruza et al. Eds. QI 2009, LNAI 5494, pp. 211-224, 2009.

Floridi, Luciano, "Semantic Conceptions of Information", The Stanford Encyclopedia of Philosophy (Spring 2015 Edition), Edward N. Zalta (ed.), forthcoming URL = http://plato.stanford.edu/archives/spr2015/entries/information-semantic/

Fodor, J., The Modularity of Mind. Cambridge, MA: MIT Press, 1983.

Gallistel, C. The Organization of Learning. MIT Press: Cambridge, MA, 1990

Goldstein, Sheldon, "Bohmian Mechanics", The Stanford Encyclopedia of Philosophy (Spring 2013 Edition), Edward N. Zalta (ed.), URL = http://plato.stanford.edu/archives/spr2013/entries/qm-bohm/

Hameroff, S., Gennaro, R. and Pylkkänen, P., HOT to DOT: A 'Deeper-Order Thought' Theory of Consciousness. In Brain, Mind and Cosmos [e-book], Trident, 2014.

Haven, E. Pilot-wave theory and financial option pricing. International J. of Theoretical Physics 44, 1957-1962, 2005.

Hiley, B.J.; Pylkkänen, P. Can mind affect matter via active information? Mind and Matter 2005, 3, 2, 
7-26. URL $=<$ http://www.mindmatter.de/mmpdf/hileywww.pdf $>$.

Humphreys, N., Consciousness Regained. Oxford: Oxford University Press, 1982.

Khrennikov, A. Information Dynamics in Cognitive, Psychological and Anomalous Phenomena. Series Fundamental Theories of Physics. V. 138. Kluwer, Dordrecht, 2004.

Ladyman, J.; Ross. D., Every Thing Must Go. Metaphysics Naturalized. Oxford University Press: Oxford, 2007

Libet, B. Unconscious cerebral initiative and the role of conscious will in voluntary action. Behavioral and Brain Sciences 1985, 8, 529-66.

Lorenz, K. Behind the Mirror (Rückseite dyes Speigels). Translated by R. Taylor. New York: Harcourt Brace Jovanovich: New York, 1977.

Maleeh, R. and Amani, P., Bohm's theory of the relationship of mind and matter revisited.

Neuroquantology 2012, 10, 150-163.

Maroney, O. Information and Entropy in Quantum theory. Ph D thesis, Birkbeck College, University of London. http://www.bbk.ac.uk/tpru/OwenMaroney/thesis/thesis.html, 2002.

Maroney, O. and Hiley, B.J., Quantum State Teleportation Understood Through the Bohm Interpretation, Foundations of Physics, Vol . 29, No. 9, pp. 1403-1415, 1999.

Nagel, T. Dualism. In The Oxford Companion to Philosophy. T. Honderich Ed.; Oxford University Press: Oxford and New York, 2005.

Ney, A. and Albert, D. eds., The Wave Function: Essays on the Metaphysics of Quantum Mechanics. Oxford University Press. 2013.

Oizumi, M., Albantakis, L. and Tononi, G.. From the phenomenology to the mechanisms of consciousness: integrated information theory 3.0. PLoS Comput Biol 10 (5): e1003588, 2014

Penfield, W., The Mystery of the Mind: a Critical Study of Consciousness and the Human Brain. 
Princeton, NJ: Princeton University Press, 1975.

Plato, The Phaedo. In The Dialogues of Plato. Trans. B. Jowett. Random House, New York, 1892.

Pockett, S., Banks, W. and Gallagher, S. Eds., Does Consciousness Cause Behaviour? Cambridge, MA: MIT Press, 2006.

Pylkkänen, P., Mind, Matter and the Implicate Order. Springer Frontiers Collection: Heidelberg and New York, 2007.

Revonsuo, A., Inner Presence: Consciousness as a Biological Phenomenon, Cambridge, MA: MIT Press, 2006.

Revonsuo, A., Hard to See the Problem? Journal of Consciousness Studies, 22, No. 3-4, 2015, pp. 5267

Ritchie, J., Understanding naturalism. Acumen: Durham, 2008.

Roederer, J.G., Information and Its Role in Nature; Springer: Berlin/Heidelberg, Germany, 2005.

Rosenthal, D. M., A theory of consciousness. In The Nature of Consciousness; N. Block, O. Flanagan, G. Güzeldere, Eds., MIT Press: Cambridge, MA, 1997.

Rosenthal, D. M., Concepts and definitions of consciousness. In Encyclopedia of Consciousness, ed. William P. Banks, Amsterdam: Elsevier, 2009

Searle, J., The Rediscovery of the Mind. Cambridge, Mass, MIT Press, 1992.

Shiffrin, R. and Schneider W., "Controlled and automatic processing: detection, search and attention." Psychological Review, 84: 1-64, 1977.

Smith, Q., Why cognitive scientists cannot ignore quantum mechanics? In Consciousness: New Philosophical Perspectives; Smith, Q., A. Jokic Eds.; Oxford University Press: Oxford, 2003.

Tononi, G. and Koch, C., Consciousness: Here, There but Not Everywhere. Manuscript 2014. 
Van Gulick, Robert, "Consciousness", The Stanford Encyclopedia of Philosophy (Spring 2014 Edition), Edward N. Zalta (ed.),

$\mathrm{URL}=\underline{\text { http://plato.stanford.edu/archives/spr2014/entries/consciousness/ }}$

Velmans, M., Is Human information processing conscious?, Behavioral and Brain Sciences, 14/4: 651$668,1991$.

Weber, R. Meaning as being in the implicate order philosophy of David Bohm: a conversation, in B.J. Hiley and F.D. Peat Eds. Quantum Implications: Essays in honour of David Bohm. London, Routledge, 1987. 